\title{
Textoline
}

\section{PHPWEBQUEST E CMAPTOOLS: O PAPEL DA DOCUMENTAÇÃO NO ACESSO AOS SOFTWARES}

\author{
PEREIRA, Daniervelin Renata Marques ${ }^{1}$ \\ ${ }^{1}$ Universidade Federal de Minas Gerais, Faculdade de Letras, MG, Brasil \\ * e-mail: daniervelin@yahoo.com.br
}

\begin{abstract}
RESUMO
Propomos aqui uma reflexão sobre a documentação de dois softwares educativos, Phpwebquest e CmapTools, com os objetivos de apresentar as duas ferramentas pedagógicas e, principalmente, perceber o papel desempenhado pela documentação no contexto de uso dos programas. Apresentaremos os formatos: tutorial, artigo, video-aulas, "ajuda", dentre outros e sua classificação.
\end{abstract}

Palavras-chave: documentação; softwares educativos; ensino de línguas

\section{RESUMÉ}

Nous proposons ici une réflexion sur la documentation de deux logiciels éducatifs, Phpwebquest et CmapTools, visant à présenter les deux outils d'enseignement et, surtout, de comprendre le rôle de la documentation en relation avec l'usage des programs. On ira présenter les formats: tutorial, article, leçons vidéo, "aide", entre autres, et leur classification.

Mot-clés: documentation; logiciels éducatifs, enseignement des langues

\section{Introdução}

Vários softwares educativos estão se popularizando com a ajuda de vasta documentação sobre os programas e pelo fato desta documentação estar traduzida para várias línguas. Dois exemplos de software abordados aqui são o CmapTools e o PHPWebQuest que são usados na educação, sendo o primeiro um software proprietário com a possibilidade de instalação tanto no sistema operacional Windows como no Linux e o segundo um programa em Software Livre sem a necessidade de instalação, uma vez que as produções dos usuários são hospedadas no servidor do EscolarBr, um portal dedicado à pesquisa e divulgação de ferramentas em software que podem ser usadas gratuitamente por professores e alunos. ${ }^{\mathrm{i}}$

Antes de iniciarmos a discussão sobre esses programas, descreveremos sucintamente abaixo as duas ferramentas pedagógicas criadas por meio deles: as WebQuests e os Mapas Conceituais, respectivamente.

A WebQuest é um método de pesquisa baseado em informações disponibilizadas na Internet. Processo proposto por Bernie Dodge em 1995, ele visa à investigação de algum tema de interesse por meio de um "caminho" facilitador definido previamente por um orientador. Este último fornece alguns pontos de partida, como uma introdução, as fontes de pesquisa e outras formas de orientação. Em seguida, o aluno é livre para buscar suas informações no espaço digital de forma criativa e colaborativa. Dessa forma, colaboração e cooperação são palavras-chave para uma boa WebQuest que, mais do que uma tarefa escolar, é um meio de compartilhar conhecimentos diversos a um público maior. Como atividade cognitiva, a WebQuest traz benefícios como a capacidade de integração de informações pelo aluno que deve acessar diversas fontes e devolver sua tarefa de forma clara e condensada, ou 


\section{Textoline}

seja, ele deve ser "antropofágico": devorar o que encontrar e devolver sua própria criação, refletindo a aquisição de novos conhecimentos.

Essa tarefa pressupõe um processo para seu cumprimento. Em uma homepage, o orientador disponibiliza os passos para a pesquisa, que devem conter as seguintes partes:

realizada(s) pelos alunos;

- $\quad$ uma introdução;

- $\quad$ a descrição da(s) tarefa(s) a ser(em)

- $\quad$ o processo, ou seja, um conjunto de informações que orientem os alunos no seu trabalho;

- $\quad$ os recursos a serem usados, disponíveis em uma página com links para sites onde os alunos possam encontrar quase toda a informação necessária (pode estar incluído no processo); trabalhos realizados;

- . os critérios que utilizará para avaliar os

- . uma conclusão.

Abaixo, um exemplo dessa ferramenta educativa que foi criada para servir de base a professores que queiram trabalhar com os jogos educativos em suas aulas (figura 1).

Muitas outras informações podem ser encontradas no site oficial da Universidade de San Diegoi sobre a definição de WebQuest bem como de inúmeros exemplos de seu uso em diversas línguas. Um site brasileiro também é referência sobre esse tópico: é o site do Senac-SPii que também disponibiliza WebQuests sobre vários assuntos.

Também chamados de Redes Semânticas, os mapas conceituais utilizam modelos anteriores, não sendo sua ideia totalmente inovadora. A teoria a seu respeito foi desenvolvida nos anos 70 pelo pesquisador norte-americano, Joseph Novak. Para ele, o mapa conceitual é uma ferramenta para organizar e representar o conhecimento. Baseado na teoria da aprendizagem significativa de David Ausubel, Novak define mapas conceituais como uma representação gráfica em duas dimensões de um conjunto de conceitos construída de tal forma que as relações entre eles sejam evidentes. Os conceitos aparecem dentro de caixas enquanto as relações entre os conceitos são especificadas através de frases de ligação nos arcos que os unem. (DUTRA \& JOHANN, s/d, p. 1).

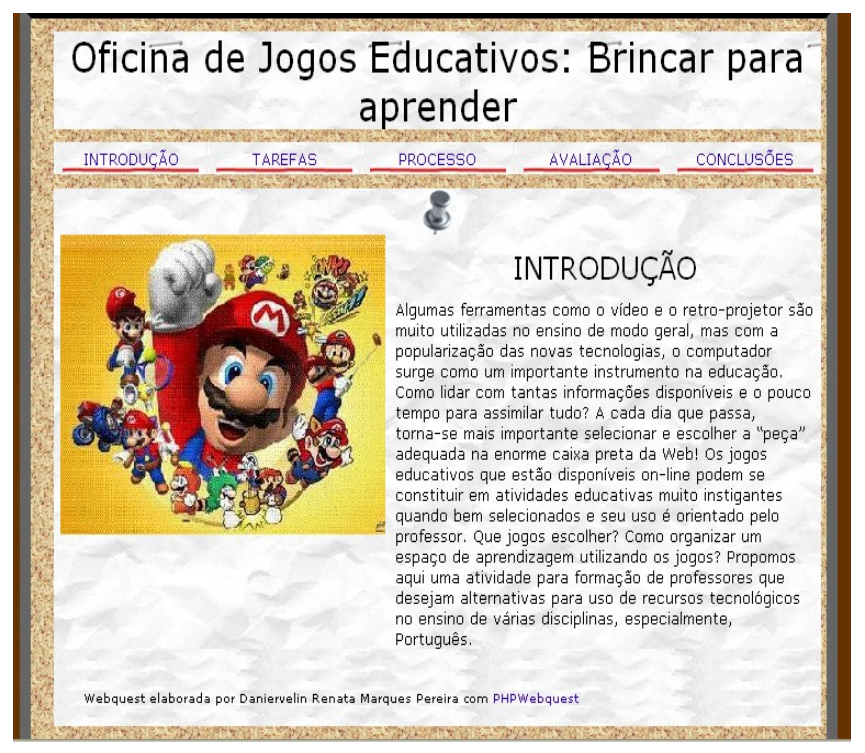

Fig. 1: WebQuesti ${ }^{i i}$ criada no programa PHPWebQuest.

Uma definição, usando a própria ferramenta citada acima, pode ser vista na figura 2 .

Nessa imagem, percebemos que os Mapas Conceituais se utilizam de frases de ligação entre conceitos organizados hierarquicamente de forma a estabelecer relações lógicas entre as ideias de um texto qualquer.

Para termos subsídios para fazer essa análise dos dois softwares citados, estabelecemos uma classificação dos tipos de documentação que encontramos em pesquisas na web.

A documentação no seu sentido geral é um conjunto de textos sobre um determinado objeto com a intenção de reunir informações relevantes sobre ele e sobre sua utilização. Podemos falar em documentação de um imóvel, de uma pessoa, de uma empresa, etc. Aqui, dedicamo-nos à documentação de softwares, a qual tem um papel fundamental de orientação aos seus usuários, uma vez que algumas informações para uso, como instalação, nem sempre são as mesmas para todos os softwares. 


\section{Textoline}

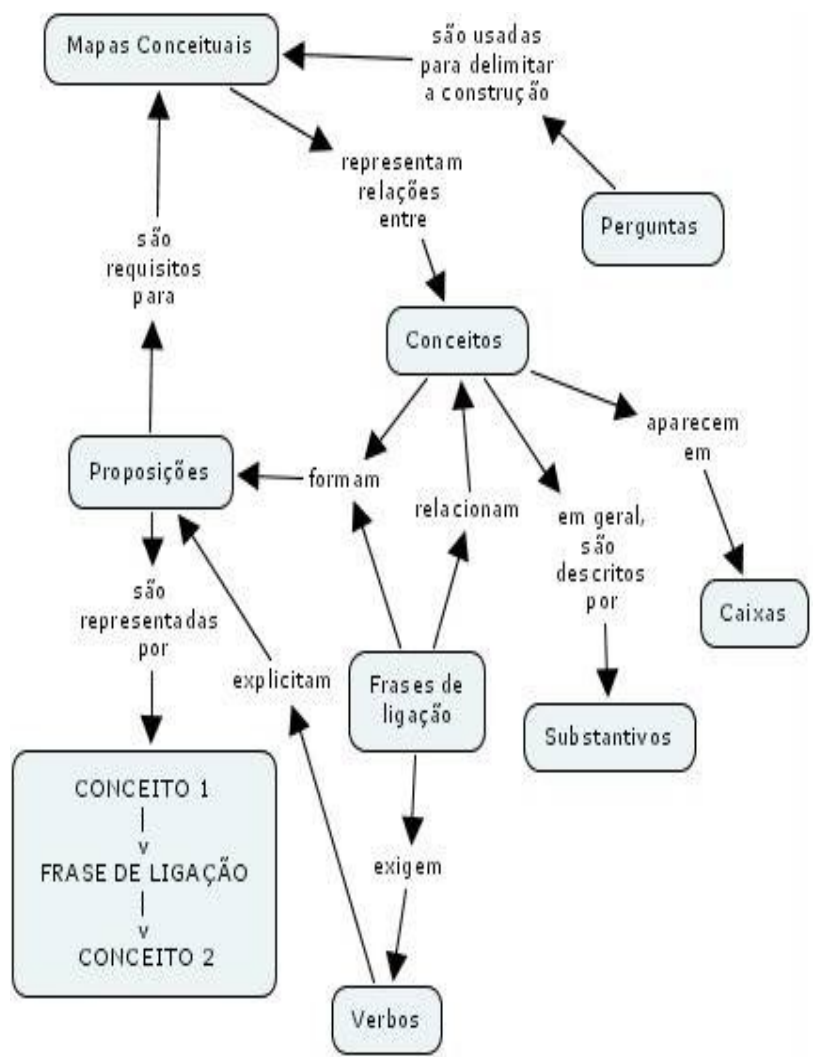

Fig. 2: O que são mapas conceituais? (DUTRA, 2005, s/p)

Os documentos de um imóvel, por exemplo, servem, em geral, para oficializarem sua existência, identificarem seu proprietário, as condições de aluguel, como deve ser usado, como e quando deve ser feita a vistoria, qual é a sua planta, além de outras informações específicas. (MATTE, 2008, s/p). Com os softwares é semelhante, tendo, em geral, um enfoque maior para a documentação que se dedica a sua instalação e uso, o que chamamos de tutorial, e para as notícias referentes a eles.

Faremos aqui uma tentativa de classificação dessa documentação para facilitar a análise dos dois programas mencionados a serem estudados aqui. Tal classificação se baseia nos tipos mais comuns de material encontrados na web sobre os softwares. Citaremos exemplos após as definições:

1) Fonte (código fonte, SourceForge e outros sites de repositório);

Código fonte ou código base, de acordo com a Wikipédia, é: "o conjunto de palavras ou símbolos escritos de forma ordenada, contendo instruções em uma das linguagens de programação existentes, de maneira lógica." (http://pt.wikipedia.org/wiki/C \%C3\%B3digo_fonte). Quando esse código é aberto, caso do software livre, é possível modificar seu conteúdo a favor do usuário. Para isso, é preciso conhecimento da linguagem de programação usada pelo criador. Em geral, há uma exigência que as mudanças feitas pelos usuários sejam compartilhadas com o objetivo de levar melhorias a todos os usuários finais.

O Sourceforge é, provavelmente, o website que hospeda o maior número de projetos de software livre e código aberto do mundo. Site: $<$ http://sourceforge.net/>.

\section{2) Tutorial;}

O tutorial é "um programa ou texto, contendo ou não imagens, que ensina passo a passo, didaticamente, como algo funciona. A palavra tutorial é derivada da palavra tutor visto que o seu objectivo é ensinar. Tutoriais são muito comuns na informática, onde são usados para ensinar como programas funcionam, e como podem ser operados por usuários iniciantes." (http://pt.wikipedia.org/wiki/Tutorial).

\section{3) Licença de software;}

Esse tipo de documentação é dedicado às condições de cópia, distribuição e modificação do original. É a forma de legalizar e estabelecer sob que direitos e deveres estão submetidos os usuários finais do programa.

\section{4) Notícia;}

Esse tipo é mais comum, mesmo porque é um gênero muito próximo do nosso cotidiano. Essas notícias englobam tipos como reportagem, artigos e outros textos para divulgação na mídia.

\section{5) Acadêmica;}

Aqui temos inseridos artigos e outros textos de divulgação acadêmica. Em comparação com o tipo anterior, podemos dizer que este tem um caráter mais restrito, uma vez que a notícia tem um público-alvo maior.

\section{6) Metalinguística;}




\section{Textoline}

Uma documentação pouco comum é a que se utiliza das próprias ferramentas, ou seja, sua própria linguagem para explicá-las. Um exemplo já foi dado acima quando citamos um Mapa Conceitual que o define.

Com base nessa classificação, acreditamos poder perceber se os programas apresentam diversidade de documentação e qual é o seu papel na comunidade de usuários e desenvolvedores.

\section{O PHPWebQuest e sua documentação: construindo tarefas colaborativas}

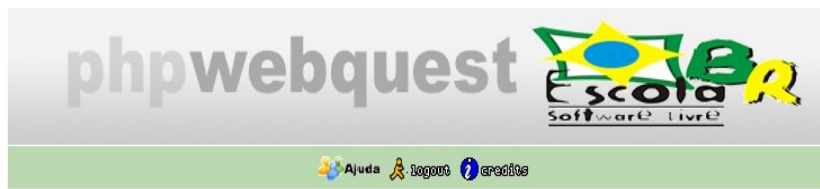

OLÁ, DANIERVELIN , seja bem-vindo ao PHPWEBQUeST do Escolabr

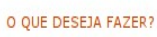

Ver, editar ou apagar alguma das minhas atividades

Criar um Webquest

Criar uma Caça ao Tesouro

Criar um Miniquest

O Solictar a inclusăo de novas Séries/Disciplina ou Área de conhecimento

Voltar ao menú principal

Fig. 3: página de criação de WebQuests: http://livre.escolabr.com/ferramentas/wq/bienvenido.php

Esse programa funciona da seguinte maneira: o usuário precisa se cadastrar no site (http://livre.escolabr.com/ferramentas/wq/) e aguardar a confirmação pelo e-mail. De posse de seu login e senha, ele está apto a criar a página da sua WebQuest facilmente. Veja as figuras 3 e 4.

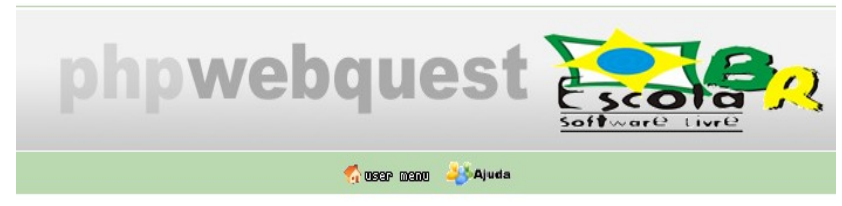

ESCOLHA UM MODELO PARA A WEBQUEST QUE DESEJA CRIAR

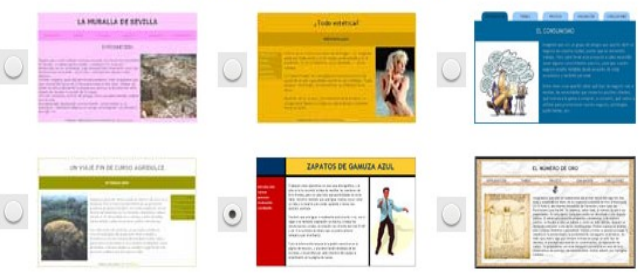

Enviar

Fig. 4: mais uma imagem de uma etapa para a criação de uma WebQuest

O programa educativo para construção de WebQuests, o PHPWebQuestiii, foi criado pelo professor espanhol Antonio Temprano e traduzido para o português pelo professor Eziquiel Menta ${ }^{\text {iv }}$ (membro do EscolaBR) para criar também Miniquest e Caças ao Tesouro sem a necessidade de escrever o código HTML ou utilizar programas de edição de páginas da web.

A documentação encontrada em língua portuguesa referente a esse software é de três tipos:

1. Fonte: código fonte e outras informações no SourceForge.net ${ }^{\mathrm{v}}$;

2. Tutorial: uma vídeo-aula;

3. Acadêmica: um artigo sobre o assunto ${ }^{\text {vi }}$.

maior parte do material sobre o programa está no blog vii em espanhol do mentor do projeto e há pouca documentação em português como pudemos ver. Isso também leva, consequentemente, a uma baixa divulgação do software. O conhecimento da existência do programa já diz tudo: foi por acaso, numa pesquisa sobre WebQuests na internet após ter feito todo um curso sobre o assunto e não ter visto nenhum dos textos mencionando o programa.

(2005) ressalta:

Entre as qualidades do software, Barros

(...) dispensa-se a necessidade do usuário encontrar um servidor, domínio, upload e demais tecnicidades. Além de podermos 


\section{Textoline}

participar propondo soluções de ordem técnica e realizando a tradução deste para o português-brasil.

O phpwebquest foi desenvolvido por Antonio Temprano da Espanha, e no início das pesquisas, descobertas e aventuras, voltadas ao estudo desta ferramenta, colaboramos (Equipe EscolaBR) para a produção e tradução para português-brasil, da $2^{\mathrm{a}}$ versão, fato apenas possível pelas possibilidades de produção colaborativa que os softwares livres oferecem, onde as fronteiras geográficas e de idioma são quebradas, permitindo a criação de comunidades de construção do conhecimento. (p. 11).

Por essa fala, percebemos que o programa ainda é recente (2004) e é fruto de um processo colaborativo e ainda em processo de construção e tradução.

No SourceForge, o projeto está disponibilizado com detalhes, inclusive com o seu código fonte.

$\mathrm{Na}$ parte dos tutoriais, a vídeo-aula ${ }^{\text {viii, }}$, disponível no site brasileiro do projeto, foi organizada pelo professor Ezequiel Menta e traz um tutorial muito detalhado para os usuários, apesar de o link para esse material se encontrar quebrado no momento desta pesquisa. No entanto, por meio de um contato com o professor, o problema foi solucionado com o envio do link correto para acesso aos arquivos.

$\mathrm{O}$ artigo acadêmico foi elaborado por Gílian Cristina Barros (2005) e apresenta uma reflexão sobre o uso das WebQuests como uma mudança metodológica para uma aprendizagem colaborativa dos alunos. A autora relata suas dificuldades na criação dessa ferramenta até o surgimento do PHPWebQuest que facilitou o trabalho do professor.

Outras instituições educativas adotaram o projeto, como foi possível perceber em buscas na web. A Escola Secundária Dr. Francisco Fernandes Lopes de Portugal, por exemplo, disponibilizou na internet um tutorial $^{\mathrm{ix}} \mathrm{em}$ formato de vídeo muito prático que pode ser considerado uma documentação para iniciantes no uso do programa.

Entretanto, a documentação sobre o projeto no Brasil se resume às três referências citadas acima. Sobre a qualidade dessa documentação, podemos dizer que ela facilita bastante, apesar de pouca, porque o software não precisa ser instalado e é auto-explicativo no processo de uso.

\section{O CmapTools e sua documentação: construindo mapas conceituais}

O programa CmapTools ${ }^{\mathrm{x}}$ foi desenvolvido pelo Institute for Human \& Machine Cognition (IHMC) Universidade do Oeste da Flórida - para construção de Mapas Conceituais. O programa proporciona uma edição personalizada dos conceitos e suas frases de ligação. É possível criar links nos conceitos para endereços da web, para imagens ou mesmo para outro mapa conceitual, numa rede essencialmente hipertextual.

O CmapTools deve ser instalado no computador do usuário tendo como opções versões para Windows, MAC OS X, Linux e Solaris. Após a construção, ele pode compartilhar sua produção carregando seu mapa para um servidor próprio ou, caso utilize o CmapServer, utilizar o servidor do projeto e permitir o acesso de outros usuários e mesmo a colaboração síncrona entre eles.

A documentação desse programa educacional é vasta. No site oficial do projeto, ela está em inglês, mas muitos textos podem ser encontrados em português em outras fontes. Um recurso muito usado no site é produzir documentação utilizando os mapas conceituais, ou seja, um recurso metalinguístico para explicar a ferramenta fazendo uso de sua própria linguagem.

Sendo assim, a documentação encontrada, a ser detalhada a seguir, foi:

HTML);

1. Tutorial (vídeo-aula, arquivos em

2. Licença;

3. Notícia (revistas e jornais);

4. Acadêmica (artigos);

5. Metalinguística (mapas conceituais).

No site do instituto responsável pela criação do programa, podemos encontrar inúmeros textos que servem de suporte, como publicações acadêmicas, notícias publicadas em revistas, tutoriais para instalação 


\section{Textoline}

em cada sistema, incluindo um texto técnico detalhado sobre o funcionamento do software: "The Network Architecture of CmapTools" (CAÑAS et al., 2003). Ainda podem ser encontrados um tutorial detalhado no link "suporte", a licença de uso e vários mapas conceituais auto-explicativos.

Em língua portuguesa, há várias páginas com o passo-a-passo da instalação e sobre o uso do programa organizadas pela UFRGS ${ }^{\mathrm{xi}}$, contendo muitas imagens de cada etapa. Uma vídeo-aula ${ }^{\text {xii }}$ detalhada foi organizada por René de Paula JR que faz uma demonstração de uso da ferramenta. Outro tutorial também organizado pelos professores da UFRGS, apesar de mais antigo, está no Dicas On-line ${ }^{\text {xiii }}$. Ainda pode ser encontrada uma notícia no site Utilidades ${ }^{\text {xiv }}$.

Para a instalação no Linux Educacional, Rafael Nink de Carvalho disponibilizou um arquivo em $\mathrm{PDF}^{\mathrm{xv}}$ bem organizado e detalhado com imagens do processo.

\section{Outros tutoriais são:}

1) Como criar Mapas Conceituais utilizando o CmapTools por Cabral (2003) da Universidade Luterana do Brasil. (Disponível em: http://guaiba.ulbra.tche.br/ yanzer/Manual Cmap Too ls.pdf).

2) Apostila de CmapTools 3.4 organizada pelo Instituto Presbiteriano Mackenzie. (Disponível em http://www.pucsp.br/pensamentomatematico/apostilac maps.doc).

3) "CmapTools no Linux: Obtendo e instalando" está em um portal bem conhecido pelos usuários de Linux e, como o próprio título sugere, ajuda na instalação. (Disponível em: http://www.vivaolinux.com.br/artigo/CmapTools-

Gestao-do-Conhecimento-atraves-de-MapasConceituais-no-Linux/?pagina $=3$ )

4) Marinho (2008) criou um Manual em formato de slides para seus alunos da Pós-Graduação da PUC-MG e o disponibilizou no Scribd, um site de compartilhamento de documentos na web. (Disponível em: http://www.scribd.com/doc/2620257/Manual-doCmapTools-Parte-1).

Vários outros links podem ser encontrados além dos aqui citados, pois, como já foi dito, o projeto conta com um número muito grande de usuários, principalmente em universidades, onde são geradas muitas produções acadêmicas sobre a ferramenta.

Em geral, a documentação, além de vasta, é de boa qualidade e tenta atender dar suporte a diversas dúvidas tanto na instalação como no uso do software.

\section{Considerações finais}

Como foi possível perceber, a circulação de um software e sua aprovação, principalmente pela academia, contribui fortemente para um intenso uso aliado a uma extensa produção sobre o assunto em relação de consequência. Enquanto o PHPWebQuest apresenta três tipos de documentação com poucos exemplos de cada, o CmapTools apresenta cinco com vários exemplos. A quantidade faz diferença? Acreditamos que sim, pois pensamos que certamente isso tem reflexos na segurança e credibilidade do software escolhido, mas a qualidade da documentação, sem dúvidas, é tão ou mais relevante para essa recepção.

Lembramos aqui de uma crítica recente feita à tradução de um software educativo para crianças que provocou seu rebaixamento perante a sociedade. Segue um trecho: 


\section{Textoline}

Erros na tradução: GCompris criticado na imprensa portuguesa

"Devido aos erros na tradução disponibilizada aos alunos, o software educacional GCompris (gcompris.net) foi notícia central nos principais jornais e televisões em Portugal, neste fim-desemana. O semanário Expresso, um dos mais respeitados, escreve "Há frases mal construídas, outras que começam na segunda pessoa do singular e continuam na terceira (tratam o leitor por tu e por você), expressões absurdas e frases que simplesmente não fazem sentido". A notícia tem o título "Festival de asneiras no Magalhães (http://aeiou.expresso.pt/o festival de asn eiras do magalhaes=f501580)" "xvi

Essa lamentável notícia só vem reforçar nossa afirmação de que a documentação tem uma grande importância para a divulgação do software. Mesmo que ele fosse muito bom, ainda assim ele não seria aceito, pois no contexto educativo um software com inadequações quanto à norma culta não serviria aos propósitos de formação do ensino.

A discussão sobre a influência da documentação na divulgação dos softwares precisaria ainda de outras informações para ficar completa, como, por exemplo, a percepção dos usuários sobre o uso da documentação e o papel dela para eles no incentivo e facilitação na utilização do software propriamente dito. No entanto, restringimo-nos aqui a uma reflexão sobre o material encontrado observando sua quantidade e potencialidade de ajudar realmente aos usuários.

Se pensarmos que essa documentação, mais do que ajudar no uso do programa, tem o papel de "constituição" dele, podemos afirmar que sua quantidade e qualidade determinam sua existência no contexto da educação, uma vez que há inúmeros projetos como o PHPWebQuest e o CmapTools. Mesmo sendo softwares de qualidade, eles precisam de um conjunto de vários tipos de documentação necessários para dar suporte ao usuário, levando a sua difusão entre, principalmente, professores e alunos. Daí a real importância da documentação sobre os softwares em geral.

\section{REFERÊNCIAS BIBLIOGRÁFICAS:}

BARROS, Gílian Cristina. Webquest: metodologia que ultrapassa os limites do ciberespaço. In: EscolaBR Software Livre. Disponível em: $<$ http://www.gilian.escolabr.com/textos/webquest gilia ncris.pdf $>$. Acesso em 08 nov. 2008.

CAÑAS et al. The Network Architecture of CmapTools. In: Web site of IHMC ( Institute for Human \& Machine Cognition), 2003.

CABRAL, Anderson Ricardo Yanzer. Como criar Mapas Conceituais utilizando o CmapTools. Universidade Luterana do Brasil - ULBRA Guaíba. Disponível em: http://guaiba.ulbra.tche.br/ yanzer/Manual Cmap Too ls.pdf. 2003.

CARVALHO, Rafael Nink de. Manual de Instalação do CmapTools no Linux Educacional 1.0. Disponível em: $\quad$ http://rafaelnink.com/blog/wpcontent/uploads/2007/10/manual instalacao cmaptools .pdf. s/d.

DUTRA, Ítalo Modesto \& JOHANN, Stéfano Pupe. Por uma abordagem construtivista dos mapas conceituais. $\quad \mathrm{S} / \mathrm{D}$. Disponível em: http://www.eproinfo.mec.gov.br/webfolio/Mod81772/a rtigomapasconstrutivismo.pdf. Acesso em 04 set. 2008.

\section{INSTITUTO PRESBITERIANO MACKENZIE.} Apostila de CmapTools 3.4. Disponível em: http://www.pucsp.br/pensamentomatematico/apostilac maps.doc. 2004.

MATTE, Ana Cristina Fricke. Software e imóveis. In: Under-Linux.org, Blog da Acris. Disponível em: $<$ http://under-linux.org/blogs/acris/103-software-eimoveis.html>. Acesso em 11 nov. 2008. 


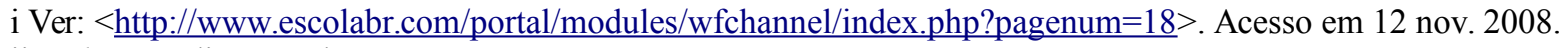

ii WebQuest disponível em:

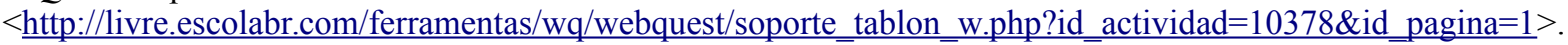

iii PHPWebQuest, software livre disponível em: $<$ http://livre.escolabr.com/ferramentas/wq/ $>$.

iv Disponível em: http://livre.escolabr.com/ferramentas/wq/. Acesso em 14 de mar. 2009.

v Disponível em: $<$ http://sourceforge.net/projects/phpwebquest/ $>$.

vi Disponível em: $<$ http://www.gilian.escolabr.com/textos/webquest giliancris.pdf $>$.

vii Disponível em: $<$ http://www.phpwebquest.org/ $>$. Acesso em 14 de mar. 2009.

viii Disponível em: $<$ http://livre.escolabr.com/ferramentas/wq/ajuda/ $>$.

ix Disponível em: $<$ http://www.projectos.esffl.pt/phpwebquest/tutorial/webquestsfim.htmll>. Acesso em 11 nov. 2008.

x Disponível em: $<$ http://cmap.ihmc.us/download/>.

xi Disponível em: $<$ http://ead.cap.ufrgs.br/pagina/cmap/> e

http://lead.cap.ufrgs.br/ tatiana/ mapas conceituais/tutorial/>.

xii Disponível em: $<$ http://rafaelnink.com/blog/2008/08/02/video-aulas-cmap-tools-mapas-conceituais/ $>$.

xiii Disponível em: $<\underline{\text { http://penta2.ufrgs.br/edutools/tutcmaps/tutindicecmap.htm }>\text {. }}$

xiv Disponível em: $<$ http://pt.utilidades-utiles.com/download-ihmc-cmaptools.html $>$.

xv Disponível em: $<$ http://rafaelnink.com/blog/wp-content/uploads/2007/10/manual_instalacao cmaptools.pdf $>$.

xvi Disponível em: $<$ http://br-linux.org/2009/erros-na-traducao-gcompris-criticado-na-impresa-portuguesa/ $>$. Acesso em 14 de mar. 2009. 